\title{
Exponential Stability Analysis for Genetic Regulatory Networks with Both Time-Varying and Continuous Distributed Delays
}

\author{
Lizi Yin ${ }^{1,2}$ and Yungang Liu ${ }^{1}$ \\ ${ }^{1}$ School of Control Science and Engineering, Shandong University, Jinan 250061, China \\ ${ }^{2}$ School of Mathematical Sciences, University of Jinan, Jinan 250022, China \\ Correspondence should be addressed to Yungang Liu; lygfr@sdu.edu.cn
}

Received 23 December 2013; Revised 8 March 2014; Accepted 9 March 2014; Published 6 May 2014

Academic Editor: Sanyi Tang

Copyright (c) 2014 L. Yin and Y. Liu. This is an open access article distributed under the Creative Commons Attribution License, which permits unrestricted use, distribution, and reproduction in any medium, provided the original work is properly cited.

\begin{abstract}
The global exponential stability is investigated for genetic regulatory networks with time-varying delays and continuous distributed delays. By choosing an appropriate Lyapunov-Krasovskii functional, new conditions of delay-dependent stability are obtained in the form of linear matrix inequality (LMI). The lower bound of derivatives of time-varying delay is first taken into account in genetic networks stability analysis, and the main results with less conservatism are established by interactive convex combination method to estimate the upper bound of derivative function of the Lyapunov-Krasovskii functional. In addition, two numerical examples are provided to illustrate the effectiveness of the theoretical results.
\end{abstract}

\section{Introduction}

From the late 20th century to the early 21st century, more than a decade's time, life science, especially molecular biology science, had great surprising changes. The research of genetic regulatory networks has become an important area in the molecular biology science and received great attention. There are plenty of results $[1,2]$. Genetic regulatory networks can be seen as biochemically dynamical systems, and it is natural to simulate them by using dynamical system model. There are a variety of models that have been proposed but mainly are the Boolean network model (discrete model) [3-6] and the differential equation model (continuous model) $[7,8]$. In the Boolean models, each gene's activity is expressed with ON or OFF, and each gene's state is described by the Boolean function of other related genes' states. In differential equation model, the variables delineate the concentrations of gene products, such as mRNAs and proteins, which are continuous values. According to a large number of biological experiments, we know that gene expression is usually continuously variable, so it is more reasonable to describe genetic regulation networks with differential equation model than with the Boolean network model.

Gene expression is a complex process regulation by the stimulation and inhibition of protein including transcription, translation, and posttranslation processes, and a large number of reactions and reacting species participate in this process. There are fast reaction and slow reaction in real genetic regulatory systems. The fast reaction includes dimerization, binding reaction, and phosphorylation, and the slow reaction contains transcription, translation, and translocation or the finite switching speed of amplifiers. Due to the slow reaction, time delays exist in genetic regulatory networks. In [9], there is a biochemistry experiment on mice which has proved that there exists a time lag of about $15 \mathrm{~min}$ in the peaks between the mRNA molecules and the proteins of the gene Hes1.

The emergence of the time delays will influence the genetic regulatory networks' dynamic behaviors, which cause the researchers' interest. The stability is one of the very important dynamic characteristics. Hence, it is necessary 
to consider the stability of genetic regulatory networks with time delay; see [10-16]. In [11], random time delays are taken into account, and some stability criteria for the uncertain delayed genetic networks with SUM regulatory logic where each transcription factor acts additively to regulate a gene were obtained; asymptotical stability criteria were proposed for genetic regulatory networks with interval time-varying delays and nonlinear disturbance in [12]. In [14], authors gave some stochastic asymptotic stability sufficient conditions for the uncertain stochastic genetic regulatory networks with both mixed timevarying delays by constructing the Lyapunov functional and employing stochastic analysis methods. In $[15,16]$, the authors studied genetic regulatory networks with constant delay.

Motivated by the above discussions, we analyze the exponential stability of genetic regulatory networks with time-varying delays and continuous distributed delays. It is worth mentioning that the asymptotical stability of genetic regulatory networks is studied in most literature; see $[17,18]$. But the exponential stability of our research is of better stability than asymptotical stability. Literature [11] discusses the exponential stability of genetic regulatory networks with random time delays, which are essentially constant delays. Our paper considers the system with interval time-varying delays and continuous distributed delays, which is more reasonable than literature [11]. Literature [19] studies robust exponential stability for stochastic genetic regulatory networks with timevarying delays, whose derivatives' upper bound is less than 1. In our models, the derivatives' upper bound of interval time-varying delays has no limit of less than 1 . And we study the lower bound of derivatives of time-varying delay to systems stability effect for the first time. In the theorem for evidence, convex combination and interactive convex combination method were adopted, which have less conservatism.

This paper is organized as follows. In Section 2, model description and some assumptions are given. In Section 3, several sufficient results are obtained to check the exponential stability for genetic regulatory networks with time-varying delays and continuous distributed delays. Some numerical examples are given to demonstrate the effectiveness of our analysis in Section 4. Finally, conclusions are drawn in Section 5 .

Notations. Throughout this paper, $\mathbf{R}, \mathbf{R}^{n}$, and $\mathbf{R}^{n \times m}$ denote, respectively, the set of all real numbers, real $n$-dimensional space, and real $n \times m$-dimensional space. $\mathbf{Z}_{+}$denote the set of all positive integers. $\|\cdot\|$ denote the Euclidean norms in $\mathbf{R}^{n}$. I and 0 denote, respectively, the identity matrix and the zero matrix with appropriate dimension. For a vector or matrix $A, A^{\mathrm{T}}$ denotes its transpose. For a square matrix $A$, $\lambda_{\max }(A)$ and $\lambda_{\text {min }}(A)$ denote the maximum eigenvalue and minimum eigenvalue of matrix $A$, respectively, and $\operatorname{sym}(A)$ is used to represent $A+A^{\mathrm{T}}$. For simplicity, in symmetric block matrices, we often use $*$ to represent the term that is induced by symmetry.

\section{Problem Formulation and Some Preliminaries}

In this paper, we are devoted to studying the stability to an autoregulatory genetic network with time delays described by the following delay differential equations:

$$
\begin{array}{r}
\dot{m}_{i}(t)=-a_{i} m_{i}(t)+\omega_{i}\left(p_{1}(t-\sigma(t)) p_{n}(t-\sigma(t))\right), \\
i=1, \ldots, n, \\
\dot{p}_{i}(t)=-c_{i} p_{i}(t)+d_{i} m_{i}(t-\tau(t)), \quad i=1, \ldots, n,
\end{array}
$$

where $m_{i}(t)$ 's and $p_{i}(t)$ 's are the concentrations of mRNAs and proteins, respectively; $a_{i}$ 's and $c_{i}$ 's are the degradation rates of mRNAs and proteins, respectively; $d_{i}$ 's are the translation rates of proteins; $\omega_{i}(\cdot)$ 's are the regulatory functions of mRNAs, being generally monotonic to each argument; and $\sigma(t)$ and $\tau(t)$ are time-varying delays.

Assumption 1. $\sigma(t)$ and $\tau(t)$ are the time-varying delay satisfying

$$
\begin{array}{cc}
0 \leq \sigma_{1} \leq \sigma(t) \leq \sigma_{2}, & \sigma_{3} \leq \dot{\sigma}(t) \leq \sigma_{4}<\infty, \\
0 \leq \tau_{1} \leq \tau(t) \leq \tau_{2}, & \tau_{3} \leq \dot{\tau}(t) \leq \tau_{4}<\infty,
\end{array}
$$

where $\sigma_{1}, \sigma_{2}, \sigma_{3}, \sigma_{4}, \tau_{1}, \tau_{2}, \tau_{3}, \tau_{4}$ are some constants.

Remark 2. In this paper, the lower bound of derivatives of time-varying delay is considered for the first time in the research of genetic regulatory networks. When information on lower bound of time-varying delay's derivatives can be measured, our results are better than the previous works.

In genetic regulatory networks, some genes can be activated by one of a few different transcription factors ("OR" logic), and others can be activated by two or more transcription factors which must be bounded at the same time ("AND" logic). In this paper, we take a model of genetic regulatory networks where each transcription factor acts additively to regulate the $i$ th gene ("SUM" logic) [20]. The regulatory function takes the form $\omega_{i}\left(p_{1}(t), \ldots, p_{n}(t)\right)=$ $\sum_{j=1}^{n} \omega_{i j}\left(p_{j}(t)\right)$, and $\omega_{i j}\left(p_{j}(t)\right)$ is a monotonic function with the following Hill form [21]:

$$
\omega_{i j}\left(p_{j}(t)\right)=\left\{\begin{array}{c}
\alpha_{i j} \frac{\left(p_{j}(t) / \beta_{j}\right)^{H_{j}}}{1+\left(p_{j}(t) / \beta_{j}\right)^{H_{j}}} \\
\text { if transcription factor } j \text { is an } \\
\text { activator of gene } i, \\
\alpha_{i j} \frac{1}{1+\left(p_{j}(t) / \beta_{j}\right)^{H_{j}}} \\
\text { if transcription factor } j \text { is a } \\
\text { repressor of gene } i,
\end{array}\right.
$$


where $H_{j}$ is the Hill coefficient, $\beta_{j}$ is a positive constant, and $\alpha_{i j}$ is the constant transcriptional rate of $j$ th transcriptional factor to $i$ th gene.

Therefore, (1) can be rewritten as

$$
\begin{array}{r}
\dot{m}_{i}(t)=-a_{i} m_{i}(t)+\sum_{j=1}^{n} b_{i j} f_{j}\left(p_{j}(t-\sigma(t))\right)+e_{i}, \\
i=1, \ldots, n, \\
\dot{p}_{i}(t)=-c_{i} p_{i}(t)+d_{i} m_{i}(t-\tau(t)), \quad i=1, \ldots, n,
\end{array}
$$

where $f_{j}(x)=\left(x / \beta_{j}\right)^{H_{j}} /\left(1+\left(x / \beta_{j}\right)\right)^{H_{j}}$ is monotonically increasing function; $e_{i}$ 's are basal rate defined by $e_{i}=\sum_{j \in U_{k}} \alpha_{i j}$ with $U_{k}=\{j \mid$ the $j$ th transcription factor being a repressor of the kth gene, $j=1, \ldots, n\}$; and matrix $B=\left(b_{i j}\right) \in \mathbf{R}^{n \times n}$ is defined as

$$
b_{i j}=\left\{\begin{array}{l}
\alpha_{i j}, \\
\quad \text { if transcription factor } j \text { is an activator } \\
\quad \text { of gene } i, \\
\quad \text { if there is no link from node } j \text { to node } i, \\
-\alpha_{i j}, \\
\quad \text { if transcription factor } j \text { is a repressor } \\
\quad \text { of gene } i .
\end{array}\right.
$$

In the compact matrix form, (4) can be rewritten as

$$
\begin{gathered}
\dot{m}(t)=-A m(t)+B f(p(t-\sigma(t)))+E, \\
\dot{p}(t)=-C P(t)+D m(t-\tau(t)),
\end{gathered}
$$

where

$$
\begin{gathered}
m(t)=\left[m_{1}(t), \ldots, m_{n}(t)\right]^{T}, \\
p(t)=\left[p_{1}(t), \ldots, p_{n}(t)\right]^{T}, \\
f(p(t))=\left[f_{1}\left(p_{1}(t)\right), \ldots, f_{n}\left(p_{n}(t)\right)\right]^{T}, \\
A=\operatorname{diag}\left(a_{1}, \ldots, a_{n}\right), \quad I=\left[e_{1}, \ldots, e_{n}\right]^{T}, \\
C=\operatorname{diag}\left(c_{1}, \ldots, c_{n}\right), \quad D=\operatorname{diag}\left(d_{1}, \ldots, d_{n}\right) .
\end{gathered}
$$

In order to get the stability results, the following assumption is necessarily imposed on (6).

Assumption 3. $f_{i}: \mathbf{R} \rightarrow \mathbf{R}, i=1, \ldots, n$, are monotonically increasing functions with saturation and moreover satisfy

$$
m_{i}^{-} \leq \frac{f_{i}(a)-f_{i}(b)}{a-b} \leq m_{i}^{+}, \quad \forall a, b \in \mathbf{R}, i=1, \ldots, n,
$$

where $m_{i}^{-}$and $m_{i}^{+}$are constants.

Remark 4. In the most existing literature, (8) was strengthened to $0 \leq\left(\left(f_{i}(a)-f_{i}(b)\right) /(a-b)\right) \leq l_{i}$, for all $a, b \in \mathbf{R}$, where $l_{i}$ 's are positive constant. Therefore, Assumption 1 is somewhat general and, in fact, similar to that of [12]; see the discussion below.
The vectors $m^{*}, p^{*}$ are said to be an equilibrium point of system (6), if they satisfy

$$
\begin{gathered}
0=-A m^{*}+B f\left(p^{*}(t-\sigma(t))\right)+E, \\
0=-C P^{*}+D m^{*}(t-\tau(t)) .
\end{gathered}
$$

$$
\begin{gathered}
\text { Let } x(t)=m(t)-m^{*} \text { and let } y(t)=p(t)-p^{*} \text {; we get } \\
\qquad \begin{array}{c}
\dot{x}(t)=-A x(t)+B g(y(t-\sigma(t))), \\
\dot{y}(t)=-C y(t)+D x(t-\tau(t)),
\end{array}
\end{gathered}
$$

where $x(t)=\left[x_{1}(t), \ldots, x_{n}(t)\right]^{T}, y(t)=\left[y_{1}(t), \ldots, y_{n}(t)\right]^{T}$, $g(y(t))=\left[g\left(y_{1}(t)\right), \ldots, g\left(y_{n}(t)\right)\right]^{T}$, and $g_{i}\left(y_{i}(t)\right)=f_{i}\left(y_{i}(t)+\right.$ $\left.p_{i}^{*}\right)-f_{i}\left(p_{i}^{*}\right)$.

By the definition of $g_{i}(\cdot)$, it satisfies sector condition:

$$
m_{i}^{-} \leq \frac{g_{i}(a)}{a} \leq m_{i}^{+}, \quad i=1, \ldots, n,
$$

which implies that

$$
\frac{g_{i}(a)-m_{i}^{-} a}{a} \geq 0, \quad \frac{m_{i}^{+} a-g_{i}(a)}{a} \geq 0 .
$$

Let $M_{0}=\operatorname{diag}\left(m_{1}^{-}, \ldots, m_{n}^{-}\right), M_{1}=\operatorname{diag}\left(m_{1}^{+}, \ldots, m_{n}^{+}\right)$, and $m=\max \left\{\left|m_{1}^{-}\right|, \ldots,\left|m_{n}^{-}\right|,\left|m_{1}^{+}\right|, \ldots,\left|m_{n}^{+}\right|\right\}$.

The initial condition of system (10) is assumed to be

$$
\begin{gathered}
x(t)=\varphi_{1}(t), \quad y(t)=\psi_{1}(t), \quad-\rho \leq t \leq 0, \\
\rho=\max \left\{\sigma_{2}, \tau_{2}\right\} .
\end{gathered}
$$

Based on system (10), we also consider the genetic regulatory networks with continuous distributed delays:

$$
\begin{gathered}
\dot{x}(t)=-A x(t)+B g(y(t-\sigma(t)))+W \int_{t-9(t)}^{t} g(y(s)) d s, \\
\dot{y}(t)=-C y(t)+D x(t-\tau(t)),
\end{gathered}
$$

where $0 \leq \vartheta(t) \leq \vartheta_{1}$.

For completeness, we recall the following definition and lemmas.

Definition 5. System (10) or (14) is said to be globally exponentially stable, if there exist constants $\lambda>0$ and $M \geq 1$, such that, for any initial value $z_{t_{0}}$,

$$
\|z(t)\| \leq M\left\|z_{t_{0}}\right\|_{C^{1}} e^{-\lambda\left(t-t_{0}\right)}
$$

hold, for all $t \geq 0$, where $z(t)=[x(t), y(t)]^{T}$ and $\|z(t)\|_{C^{1}}=$ $\sup _{-\rho \leq \theta \leq 0}\{\|z(t+\theta)\|,\|\dot{z}(t+\theta)\|\}$.

Lemma 6 (see [22]). For any positive definite matrix $M \in$ $\mathbf{R}^{n \times n}$, there exists a scalar $q>0$ and a vector-valued function $\omega:[0, q] \rightarrow \mathbf{R}^{n}$ such that

$$
\left(\int_{0}^{q} \omega(s) d s\right)^{T} M\left(\int_{0}^{q} \omega(s) d s\right) \leq q \int_{0}^{q} \omega^{T}(s) M \omega(s) d s .
$$


Lemma 7 (see [23]). Let $h_{1}, \ldots, h_{N}: \quad \mathbf{R}^{m} \rightarrow \mathbf{R}$ take positive values in an open subset $\mathbf{D}$ of $\mathbf{R}^{m}$. Then, the reciprocally convex combination of $h_{i}$ over $\mathbf{D}$ satisfies

$$
\min _{\left\{\alpha_{i} \mid \alpha_{i}>0, \sum_{i} \alpha_{i}=1\right\}} \sum_{i} \frac{1}{\alpha_{i}} h_{i}(\eta)=\sum_{i} h_{i}(\eta)+\max _{k_{i, j}(\eta)} \sum_{i \neq j} k_{i, j}(\eta)
$$

subject to

$$
\begin{gathered}
\left\{k_{i, j}: \mathbf{R}^{m} \longmapsto \mathbf{R}, k_{j, i}(\eta)=k_{i, j}(\eta),\right. \\
\left.\left[\begin{array}{cc}
h_{i}(\eta) & k_{i, j}(\eta) \\
k_{i, j}(\eta) & h_{j}(\eta)
\end{array}\right] \geq 0\right\} .
\end{gathered}
$$

\section{Main Results}

In this section, several theorems are presented of genetic regulatory networks with both time-varying delays and continuous distributed delays. Firstly, a globally exponential stability result is developed for the genetic regulatory network with time-varying delays.

Theorem 8. For system (10) with Assumptions 1 and 3, the equilibrium point is globally exponentially stable (that is, there are two positive constants $\alpha$ and $\lambda$ such that $\|z(t)\| \leq$ $\alpha e^{-\lambda t}\left\|z\left(t_{0}\right)\right\|_{C_{1}}$, for all $t \geq t_{0}$ ) if there exist positive definite matrices $H_{2}, H_{4}, P_{1}, P_{2}, Q_{i}, i=1, \ldots, 7, W_{i}, i=1, \ldots, 4, \Gamma_{1}=$ $\operatorname{diag}\left(\gamma_{11}, \ldots, \gamma_{1 n}\right)$, and $\Gamma_{2}=\operatorname{diag}\left(\gamma_{21}, \gamma_{22}, \ldots, \gamma_{2 n}\right)$, such that, for any appropriate dimensions constant matrices $H_{1}, H_{3}, X, Y$, the following LMIs hold:

$$
\begin{gathered}
\left(\begin{array}{cc}
W_{2} & X \\
X & W_{2}
\end{array}\right) \geq 0, \quad\left(\begin{array}{cc}
W_{4} & Y \\
Y & W_{4}
\end{array}\right) \geq 0, \\
\Omega_{1}+\Omega_{2}+O_{3}^{T}\left[e^{-\lambda \tau_{2}} Q_{2}+e^{-\lambda \tau_{1}} Q_{3}\right] O_{3} \\
+O_{8}^{T}\left[e^{-\lambda \sigma_{2}} Q_{5}+e^{-\lambda \sigma_{1}} Q_{6}\right] O_{8}<0,
\end{gathered}
$$

where $\Omega_{1}=\operatorname{diag}\left(\lambda P_{1}+Q_{1}-e^{-\lambda \tau_{1}} W_{1}-H_{1}^{T} A-A^{T} H_{1}\right.$, $e^{-\lambda \tau_{1}}\left(Q_{1}-Q_{2}\right)-e^{-\lambda \tau_{1}} W_{1}-e^{-\lambda \tau_{2}} W_{2}, e^{-\lambda \tau_{1}}\left(1-\tau_{4}\right) Q_{3}-e^{-\lambda \tau_{2}}[(1-$ $\left.\left.\tau_{3}\right) Q_{2}+2 W_{2}-X^{T}-X\right],-e^{-\lambda \tau_{2}} W_{2}, \tau_{1}^{2} W_{1}+\left(\tau_{2}-\tau_{1}\right)^{2} W_{2}-H_{2}^{T}-H_{2}$, $\lambda P_{2}+Q_{4}-e^{-\lambda \sigma_{1}} W_{3}-2 M_{1}^{T} \Gamma_{1} M_{0}-H_{3}^{T} C-C^{T} H_{3},-e^{-\lambda \sigma_{1}}\left(Q_{4}-Q_{5}+\right.$ $\left.W_{3}\right)-e^{-\lambda \sigma_{2}} W_{4}, e^{-\lambda \sigma_{1}}\left(1-\sigma_{4}\right) Q_{6}-e^{-\lambda \sigma_{2}}\left[\left(1-\sigma_{3}\right) Q_{5}+2 W_{4}-Y^{T}-\right.$ $Y]-M_{1}^{T} \Gamma_{2} M_{0},-e^{-\lambda \sigma_{2}}\left(Q_{4}+W_{4}\right), \sigma_{1}^{2} W_{3}+\left(\sigma_{2}-\sigma_{1}\right)^{2} W_{4}-H_{4}^{T}-$ $\left.H_{4}, Q_{7}-2 \Gamma_{1},-e^{-\lambda \sigma_{2}} Q_{7}\left(1-\sigma_{d}\right)-2 \Gamma_{2}\right), \Omega_{2}=\operatorname{sym}\left(O_{1}^{T} e^{-\lambda \tau_{1}} W_{1} O_{2}\right.$ $+O_{1}^{T}\left[P_{1}-H_{1}^{T}-H_{2}^{T} A\right] O_{5}+O_{1}^{T} H_{1}^{T} B O_{12}+O_{2}^{T} e^{-\lambda \tau_{2}}\left(W_{2}-\right.$ $X) O_{3}+O_{2}^{T} e^{-\lambda \tau_{2}} X_{4}+O_{3}^{T} e^{-\lambda \tau_{2}}\left(W_{2}-X\right) O_{4}+O_{3}^{T} H_{3}^{T} D O_{6}+$ $O_{3}^{T} H_{4}^{T} D O_{10}+O_{5}^{T} H_{2}^{T} B O_{12}+O_{6}^{T} e^{-\lambda \sigma_{1}} W_{3} O_{7}+O_{6}^{T}\left[P_{2}-H_{3}^{T}-\right.$ $\left.H_{4}^{T} C\right] O_{10}+O_{6}^{T}\left[\Gamma_{1} M_{0}+M_{1}^{T} \Gamma_{1}\right] O_{11}+O_{7}^{T} e^{-\lambda \sigma_{2}}\left(W_{4}-Y\right) O_{8}+$ $\left.O_{7}^{T} e^{-\lambda \sigma_{2}} Y O_{9}+O_{8}^{T} e^{-\lambda \sigma_{2}}\left(W_{4}-Y\right) O_{9}+O_{8}^{T}\left[\Gamma_{2} M_{0}+M_{1}^{T} \Gamma_{2}\right] O_{12}\right)$, and $O_{i}=\left[0_{n \times(i-1) n}, I_{n \times n}, 0_{n \times(13-i) n}\right], i=1, \ldots, 12$.
Proof. Based on system (10), we construct the following Lyapunov-Krasovskii functional:

$$
\begin{aligned}
V(x(t), y(t))= & V_{1}(x(t), y(t))+V_{2}(x(t), y(t)) \\
& +V_{3}(x(t), y(t)),
\end{aligned}
$$

where

$$
\begin{aligned}
& V_{1}(x(t), y(t))=x^{T}(t) P_{1} x(t)+y^{T}(t) P_{2} y(t), \\
& V_{2}(x(t), y(t))=\int_{t-\tau_{1}}^{t} e^{\lambda(s-t)} x^{T}(s) Q_{1} x(s) d s \\
& +\int_{t-\tau(t)}^{t-\tau_{1}} e^{\lambda(s-t)} x^{T}(s) Q_{2} x(s) d s \\
& +\int_{t-\tau_{2}}^{t-\tau(t)} e^{\lambda(s-t)} x^{T}(s) Q_{3} x(s) d s \\
& +\int_{t-\sigma_{1}}^{t} e^{\lambda(s-t)} y^{T}(s) Q_{4} y(s) d s \\
& +\int_{t-\sigma(t)}^{t-\sigma_{1}} e^{\lambda(s-t)} y^{T}(s) Q_{5} y(s) d s \\
& +\int_{t-\sigma_{2}}^{t-\sigma(t)} e^{\lambda(s-t)} y^{T}(s) Q_{6} y(s) d s \\
& +\int_{t-\sigma(t)}^{t} e^{\lambda(s-t)} g^{T}(y(s)) Q_{7} g(y(s)) d s, \\
& V_{3}(x(t), y(t)) \\
& =\int_{-\tau_{1}}^{0} \int_{t+\theta}^{t} \tau_{1} e^{\lambda(s-t)} \dot{x}^{T}(s) W_{1} \dot{x}(s) d s d \theta \\
& +\int_{-\tau_{2}}^{-\tau_{1}} \int_{t+\theta}^{t}\left(\tau_{2}-\tau_{1}\right) e^{\lambda(s-t)} \dot{x}^{T}(s) W_{2} \dot{x}(s) d s d \theta \\
& +\int_{-\sigma_{1}}^{0} \int_{t+\theta}^{t} \sigma_{1} e^{\lambda(s-t)} \dot{y}^{T}(s) W_{3} \dot{y}(s) d s d \theta \\
& +\int_{-\sigma_{2}}^{-\sigma_{1}} \int_{t+\theta}^{t}\left(\sigma_{2}-\sigma_{1}\right) e^{\lambda(s-t)} \dot{y}^{T}(s) W_{4} \dot{y}(s) d s d \theta
\end{aligned}
$$

Taking the derivatives of $V_{i}, i=1,2,3$, we have

$$
\begin{gathered}
\dot{V}_{1}(x(t), y(t))=2 x^{T}(t) P_{1} \dot{x}(t)+2 y^{T}(t) P_{2} \dot{y}(t), \\
\dot{V}_{2}(x(t), y(t)) \\
=-\lambda \int_{t-\tau_{1}}^{t} e^{\lambda(s-t)} x^{T}(s) Q_{1} x(s) d s \\
-\lambda \int_{t-\tau(t)}^{t-\tau_{1}} e^{\lambda(s-t)} x^{T}(s) Q_{2} x(s) d s \\
-\lambda \int_{t-\tau_{2}}^{t-\tau(t)} e^{\lambda(s-t)} x^{T}(s) Q_{3} x(s) d s
\end{gathered}
$$




$$
\begin{aligned}
& -\lambda \int_{t-\sigma_{1}}^{t} e^{\lambda(s-t)} y^{T}(s) Q_{4} y(s) d s \\
& -\lambda \int_{t-\sigma(t)}^{t-\sigma_{1}} e^{\lambda(s-t)} y^{T}(s) Q_{5} y(s) d s \\
& -\lambda \int_{t-\sigma_{2}}^{t-\sigma(t)} e^{\lambda(s-t)} y^{T}(s) Q_{6} y(s) d s \\
& -\lambda \int_{t-\sigma(t)}^{t} e^{\lambda(s-t)} g^{T}(y(s)) Q_{7} g(y(s)) d s \\
& +x^{T}(t) Q_{1} x(t)-e^{-\lambda \tau_{1}} x^{T}\left(t-\tau_{1}\right) Q_{1} x\left(t-\tau_{1}\right) \\
& +e^{-\lambda \tau_{1}} x^{T}\left(t-\tau_{1}\right) Q_{2} x\left(t-\tau_{1}\right) \\
& -e^{-\lambda \tau_{2}} x^{T}(t-\tau(t)) Q_{2} x(t-\tau(t))(1-\dot{\tau}(t)) \\
& -e^{-\lambda \tau_{2}} x^{T}\left(t-\tau_{2}\right) Q_{3} x\left(t-\tau_{2}\right) \\
& +e^{-\lambda \tau_{1}} x^{T}(t-\tau(t)) Q_{3} x(t-\tau(t))(1-\dot{\tau}(t)) \\
& +y^{T}(t) Q_{4} y(t)-e^{-\lambda \sigma_{1}} y^{T}\left(t-\sigma_{1}\right) Q_{4} y\left(t-\sigma_{1}\right) \\
& +e^{-\lambda \sigma_{1}} y^{T}\left(t-\sigma_{1}\right) Q_{5} y\left(t-\sigma_{1}\right) \\
& -e^{-\lambda \sigma_{2}} y^{T}(t-\sigma(t)) Q_{5} y(t-\sigma(t))(1-\dot{\sigma}(t)) \\
& -e^{-\lambda \sigma_{2}} y^{T}\left(t-\sigma_{2}\right) Q_{6} y\left(t-\sigma_{2}\right) \\
& +e^{-\lambda \sigma_{1}} y^{T}(t-\sigma(t)) Q_{6} y(t-\sigma(t))(1-\dot{\sigma}(t)) \\
& +g^{T}(y(t)) Q_{7} g(y(t))-e^{-\lambda \sigma_{2}} g^{T} \\
& \times(y(t-\sigma(t))) Q_{7} g(y(t-\sigma(t)))(1-\dot{\sigma}(t)), \\
& \dot{V}_{3}(x(t), y(t)) \\
& =-\lambda \int_{-\tau_{1}}^{0} \int_{t+\theta}^{t} \tau_{1} e^{\lambda(s-t)} \dot{x}^{T}(s) W_{1} \dot{x}(s) d s d \theta \\
& -\lambda \int_{-\tau_{2}}^{-\tau_{1}} \int_{t+\theta}^{t}\left(\tau_{2}-\tau_{1}\right) e^{\lambda(s-t)} \dot{x}^{T}(s) W_{2} \dot{x}(s) d s d \theta \\
& -\lambda \int_{-\sigma_{1}}^{0} \int_{t+\theta}^{t} \sigma_{1} e^{\lambda(s-t)} \dot{y}^{T}(s) W_{3} \dot{y}(s) d s d \theta \\
& -\lambda \int_{-\sigma_{2}}^{-\sigma_{1}} \int_{t+\theta}^{t}\left(\sigma_{2}-\sigma_{1}\right) e^{\lambda(s-t)} \dot{y}^{T}(s) W_{4} \dot{y}(s) d s d \theta \\
& +\tau_{1}^{2} \dot{x}^{T}(t) W_{1} \dot{x}(t)-\int_{t-\tau_{1}}^{t} \tau_{1} e^{\lambda(\theta-t)} \dot{x}^{T}(\theta) W_{1} \dot{x}(\theta) d \theta \\
& +\left(\tau_{2}-\tau_{1}\right)^{2} \dot{x}^{T}(t) W_{2} \dot{x}(t) \\
& -\int_{t-\tau_{2}}^{t-\tau_{1}}\left(\tau_{2}-\tau_{1}\right) e^{\lambda(\theta-t)} \dot{x}^{T}(\theta) W_{2} \dot{x}(\theta) d \theta \\
& +\sigma_{1}^{2} \dot{y}^{T}(t) W_{3} \dot{y}(t)-\int_{t-\sigma_{1}}^{t} \sigma_{1} e^{\lambda(\theta-t)} \dot{y}^{T}(\theta) W_{3} \dot{y}(\theta) d \theta
\end{aligned}
$$

$$
\begin{aligned}
& +\left(\sigma_{2}-\sigma_{1}\right)^{2} \dot{y}^{T}(t) W_{4} \dot{y}(t) \\
& -\int_{t-\sigma_{2}}^{t-\sigma_{1}}\left(\sigma_{2}-\sigma_{1}\right) e^{\lambda(\theta-t)} \dot{y}^{T}(\theta) W_{4} \dot{y}(\theta) d \theta .
\end{aligned}
$$

From Lemma 6, we have

$$
\begin{aligned}
& -\int_{t-\tau_{1}}^{t} \tau_{1} e^{\lambda(\theta-t)} \dot{x}^{T}(\theta) W_{1} \dot{x}(\theta) d \theta \\
& \quad \leq-e^{-\lambda \tau_{1}}\left[x(t)-x\left(t-\tau_{1}\right)\right]^{T} W_{1}\left[x(t)-x\left(t-\tau_{1}\right)\right], \\
& -\int_{t-\sigma_{1}}^{t} \sigma_{1} e^{\lambda(\theta-t)} \dot{y}^{T}(\theta) W_{3} \dot{y}(\theta) d \theta \\
& \quad \leq-e^{-\lambda \sigma_{1}}\left[y(t)-y\left(t-\sigma_{1}\right)\right]^{T} W_{3}\left[y(t)-y\left(t-\sigma_{1}\right)\right] .
\end{aligned}
$$

Meanwhile,

$$
\begin{aligned}
& -\int_{t-\tau_{2}}^{t-\tau_{1}}\left(\tau_{2}-\tau_{1}\right) e^{\lambda(\theta-t)} \dot{x}^{T}(\theta) W_{2} \dot{x}(\theta) d \theta \\
& \leq-e^{-\lambda \tau_{2}}\left[\int_{t-\tau_{2}}^{t-\tau(t)}\left(\tau_{2}-\tau_{1}\right) \dot{x}^{T}(\theta) W_{2} \dot{x}(\theta) d \theta\right. \\
& \left.+\int_{t-\tau(t)}^{t-\tau_{1}}\left(\tau_{2}-\tau_{1}\right) \dot{x}^{T}(\theta) W_{2} \dot{x}(\theta) d \theta\right] \\
& =-e^{-\lambda \tau_{2}}\left[\int_{t-\tau_{2}}^{t-\tau(t)}\left(\tau_{2}-\tau(t)\right) \dot{x}^{T}(\theta) W_{2} \dot{x}(\theta) d \theta\right. \\
& +\int_{t-\tau(t)}^{t-\tau_{1}}\left(\tau(t)-\tau_{1}\right) \dot{x}^{T}(\theta) W_{2} \dot{x}(\theta) d \theta \\
& +\int_{t-\tau_{2}}^{t-\tau(t)}\left(\tau(t)-\tau_{1}\right) \dot{x}^{T}(\theta) W_{2} \dot{x}(\theta) d \theta \\
& \left.+\int_{t-\tau(t)}^{t-\tau_{1}}\left(\tau_{2}-\tau(t)\right) \dot{x}^{T}(\theta) W_{2} \dot{x}(\theta) d \theta\right] \\
& =-e^{-\lambda \tau_{2}}\left[\int_{t-\tau_{2}}^{t-\tau(t)}\left(\tau_{2}-\tau(t)\right) \dot{x}^{T}(\theta) W_{2} \dot{x}(\theta) d \theta\right. \\
& +\int_{t-\tau(t)}^{t-\tau_{1}}\left(\tau(t)-\tau_{1}\right) \dot{x}^{T}(\theta) W_{2} \dot{x}(\theta) d \theta \\
& +\frac{\tau(t)-\tau_{1}}{\tau_{2}-\tau(t)} \\
& \times \int_{t-\tau_{2}}^{t-\tau(t)}\left(\tau_{2}-\tau(t)\right) \dot{x}^{T}(\theta) W_{2} \dot{x}(\theta) d \theta \\
& +\frac{\tau_{2}-\tau(t)}{\tau(t)-\tau_{1}} \\
& \left.\times \int_{t-\tau(t)}^{t-\tau_{1}}\left(\tau(t)-\tau_{1}\right) \dot{x}^{T}(\theta) W_{2} \dot{x}(\theta) d \theta\right] \text {. }
\end{aligned}
$$


By Lemma 6, we obtain that

$$
\begin{gathered}
-\int_{t-\tau_{2}}^{t-\tau_{1}}\left(\tau_{2}-\tau_{1}\right) e^{\lambda(\theta-t)} \dot{x}^{T}(\theta) W_{2} \dot{x}(\theta) d \theta \\
\leq-e^{-\lambda \tau_{2}}\left\{\frac{\tau_{2}-\tau_{1}}{\tau_{2}-\tau(t)}\left[x(t-\tau(t))-x\left(t-\tau_{2}\right)\right]^{T}\right. \\
\times W_{2}\left[x(t-\tau(t))-x\left(t-\tau_{2}\right)\right] \\
+\frac{\tau_{2}-\tau_{1}}{\tau(t)-\tau_{1}}\left[x\left(t-\tau_{1}\right)-x(t-\tau(t))\right]^{T} \\
\left.\times W_{2}\left[x\left(t-\tau_{1}\right)-x(t-\tau(t))\right]\right\} .
\end{gathered}
$$

And by Lemma 7, we get that

$$
\begin{aligned}
&-\int_{t-\tau_{2}}^{t-\tau_{1}}\left(\tau_{2}-\tau_{1}\right) e^{\lambda(\theta-t)} \dot{x}^{T}(\theta) W_{2} \dot{x}(\theta) d \theta \\
& \leq-e^{-\lambda \tau_{2}}\left\{\left[x\left(t-\tau_{1}\right)-x(t-\tau(t))\right]^{T}\right. \\
& \times W_{2}\left[x\left(t-\tau_{1}\right)-x(t-\tau(t))\right] \\
&+\left[x(t-\tau(t))-x\left(t-\tau_{2}\right)\right]^{T} \\
& \times W_{2}\left[x(t-\tau(t))-x\left(t-\tau_{2}\right)\right] \\
&+2\left[x\left(t-\tau_{1}\right)-x(t-\tau(t))\right]^{T} \\
&\left.\times X\left[x(t-\tau(t))-x\left(t-\tau_{2}\right)\right]\right\} .
\end{aligned}
$$

Similar to (27),

$$
\begin{aligned}
-\int_{t-\sigma_{2}}^{t-\sigma_{1}} e^{\lambda(\theta-t)} \dot{y}^{T}(\theta) & W_{4} \dot{y}(\theta) d \theta \\
\leq-e^{-\lambda \sigma_{2}}\{[y(t & \left.\left.-\sigma_{1}\right)-y(t-\sigma(t))\right]^{T} \\
& \times W_{4}\left[y\left(t-\sigma_{1}\right)-y(t-\sigma(t))\right] \\
& +\left[y(t-\sigma(t))-y\left(t-\sigma_{2}\right)\right]^{T} \\
& \times W_{4}\left[y(t-\sigma(t))-y\left(t-\sigma_{2}\right)\right] \\
& +2\left[y\left(t-\sigma_{1}\right)-y(t-\sigma(t))\right]^{T} \\
& \left.\times Y\left[y(t-\sigma(t))-y\left(t-\sigma_{2}\right)\right]\right\} .
\end{aligned}
$$

By Assumption 3, for any $\Gamma_{i}=\operatorname{diag}\left(\gamma_{i 1}, \ldots, \gamma_{i n}\right) \geq 0, i=1,2$, the following inequality is true:

$$
\begin{aligned}
-2 \sum_{i=1}^{n} \gamma_{i 1}\left[g_{i}\left(y_{i}(t)\right)-m_{i}^{+} y_{i}(t)\right]\left[g_{i}\left(y_{i}(t)\right)-m_{i}^{-} y_{i}(t)\right] \\
-2 \sum_{i=1}^{n} \gamma_{i 2}\left[g_{i}\left(y_{i}(t-\tau(t))\right)-m_{i}^{+} y_{i}(t-\tau(t))\right] \\
\times\left[g_{i}\left(y_{i}(t-\tau(t))\right)-m_{i}^{-} y_{i}(t-\tau(t))\right] \geq 0 .
\end{aligned}
$$

It can be rewritten as

$$
\begin{aligned}
& -2\left[g(y(t))-M_{1} y(t)\right]^{T} \Gamma_{1}\left[g(y(t))-M_{0} y(t)\right] \\
& -2\left[g(y(t-\sigma(t)))-M_{1} y(t-\sigma(t))\right]^{T} \\
& \quad \times \Gamma_{2}\left[g(y(t-\sigma(t)))-M_{0} y(t-\sigma(t))\right] \geq 0 .
\end{aligned}
$$

For any constant matrices of appropriate dimensions $H_{i}, \quad i=$ $1, \ldots, 4$, and from (10), we can obtain that

$$
\begin{aligned}
0= & 2\left[x^{T}(t) H_{1}^{T}+\dot{x}^{T}(t) H_{2}^{T}\right] \\
& \times[-\dot{x}(t)-A x(t)+B g(y(t-\sigma(t)))], \\
0= & 2\left[y^{T}(t) H_{3}^{T}+\dot{y}^{T}(t) H_{4}^{T}\right] \\
& \times[-\dot{y}(t)-C y(t)+D x(t-\tau(t))] .
\end{aligned}
$$

Combining (21)-(31), we have

$$
\begin{gathered}
\dot{V}(x(t), y(t))+\lambda V(x(t), y(t)) \\
\leq \xi^{T}(t)\left\{\Omega+O_{3}^{T}\left[e^{-\lambda \tau_{2}}\left(\dot{\tau}(t)-\tau_{3}\right) Q_{2}\right.\right. \\
\left.+e^{-\lambda \tau_{1}}\left(\tau_{4}-\dot{\tau}(t)\right) Q_{3}\right] O_{3} \\
+O_{8}^{T}\left[e^{-\lambda \sigma_{2}}\left(\dot{\sigma}(t)-\sigma_{3}\right) Q_{5}\right. \\
\left.\left.+e^{-\lambda \sigma_{1}}\left(\sigma_{4}-\dot{\sigma}(t)\right) Q_{6}\right] O_{8}\right\} \xi(t),
\end{gathered}
$$

where

$$
\begin{gathered}
\xi^{T}(t)=\left[x^{T}(t), x^{T}\left(t-\tau_{1}\right), x^{T}(t-\tau(t)), x^{T}\left(t-\tau_{2}\right),\right. \\
\dot{x}^{T}(t), y^{T}(t), y^{T}\left(t-\sigma_{1}\right), y^{T}(t-\sigma(t)), \\
\left.y^{T}\left(t-\sigma_{2}\right), \dot{y}^{T}(t), g^{T}(y(t)), g^{T}(y(t-\sigma(t)))\right] .
\end{gathered}
$$

From (19) and (20), we can see that

$$
\dot{V}(x(t), y(t))+\lambda V(x(t), y(t))<0
$$

for all nonzero $\xi(t)$. Integrating the above inequality (34) from $t_{0}$ to $t$ gives

$$
V(x(t), y(t)) \leq e^{-\lambda\left(t-t_{0}\right)} V\left(x\left(t_{0}\right), y\left(t_{0}\right)\right) .
$$

From (21), we know that

$$
V(x(t), y(t)) \geq V_{1}(x(t), y(t))
$$

$$
\geq \min \left\{\lambda_{\min }\left(P_{1}\right), \lambda_{\min }\left(P_{2}\right)\right\}\|z(t)\|^{2},
$$




$$
\begin{aligned}
& V\left(x\left(t_{0}\right), y\left(t_{0}\right)\right) \\
& \leq\left[\lambda_{\max }\left(P_{1}\right)+\lambda_{\max }\left(P_{2}\right)+\tau_{1} \lambda_{\max }\left(Q_{1}\right)\right. \\
& +\left(\tau_{2}-\tau_{1}\right)\left[\lambda_{\max }\left(Q_{2}\right)+\lambda_{\max }\left(Q_{3}\right)\right] \\
& +\left(\sigma_{2}-\sigma_{1}\right)\left[\lambda_{\max }\left(Q_{5}\right)+\lambda_{\max }\left(Q_{6}\right)\right] \\
& +\sigma_{1} \lambda_{\max }\left(Q_{4}\right)+\sigma_{2} m^{2} \lambda_{\max }\left(Q_{7}\right) \\
& +\frac{1}{2} \tau_{1}^{3} \lambda_{\max }\left(W_{1}\right)+\frac{1}{2}\left(\tau_{2}-\tau_{1}\right)^{3} \lambda_{\max }\left(W_{2}\right) \\
& \left.\quad+\frac{1}{2} \sigma_{1}^{3} \lambda_{\max }\left(W_{3}\right)+\frac{1}{2}\left(\sigma_{2}-\sigma_{1}\right)^{3} \lambda_{\max }\left(W_{4}\right)\right]\left\|z\left(t_{0}\right)\right\|_{C_{1}}^{2}
\end{aligned}
$$

Let

$$
\begin{aligned}
& \lambda_{1}=\min \left\{\lambda_{\min }\left(P_{1}\right), \lambda_{\min }\left(P_{2}\right)\right\} \\
\lambda_{2}= & \lambda_{\max }\left(P_{1}\right)+\lambda_{\max }\left(P_{2}\right)+\tau_{1} \lambda_{\max }\left(Q_{1}\right) \\
& +\left(\tau_{2}-\tau_{1}\right)\left[\lambda_{\max }\left(Q_{2}\right)+\lambda_{\max }\left(Q_{3}\right)\right] \\
& +\left(\sigma_{2}-\sigma_{1}\right)\left[\lambda_{\max }\left(Q_{5}\right)+\lambda_{\max }\left(Q_{6}\right)\right] \\
& +\sigma_{1} \lambda_{\max }\left(Q_{4}\right)+\sigma_{2} m^{2} \lambda_{\max }\left(Q_{7}\right) \\
& +\frac{1}{2} \tau_{1}^{3} \lambda_{\max }\left(W_{1}\right)+\frac{1}{2}\left(\tau_{2}-\tau_{1}\right)^{3} \lambda_{\max }\left(W_{2}\right) \\
& +\frac{1}{2} \sigma_{1}^{3} \lambda_{\max }\left(W_{3}\right)+\frac{1}{2}\left(\sigma_{2}-\sigma_{1}\right)^{3} \lambda_{\max }\left(W_{4}\right) .
\end{aligned}
$$

Then, by (35) and (36), we have

$$
\lambda_{1}\|z(t)\|^{2} \leq V(x(t), y(t)) \leq \lambda_{2} e^{-\lambda\left(t-t_{0}\right)}\left\|z\left(t_{0}\right)\right\|_{C_{1}}^{2} .
$$

By (38), we get that

$$
\|z(t)\|^{2} \leq \frac{\lambda_{2}}{\lambda_{1}} e^{-\lambda\left(t-t_{0}\right)}\left\|z\left(t_{0}\right)\right\|_{C_{1}}^{2}
$$

Let $\alpha=\left(\lambda_{2} / \lambda_{1}\right)^{1 / 2}$, and, by Definition 5 , the genetic regulatory networks in (10) are exponentially stable.

The proof is completed.

In the following, we consider the globally exponential stability of the genetic regulatory networks with time-varying delays and continuous distributed delays.

Theorem 9. For system (14) with Assumptions 1 and 3, the equilibrium point is globally exponentially stable (that is, there are two positive constants $\alpha^{\prime}$ and $\lambda$ such that $\|z(t)\| \leq$ $\alpha e^{-\lambda t}\left\|z\left(t_{0}\right)\right\|_{C_{1}}$, for all $\left.t \geq t_{0}\right)$ if there exist positive definite matrices $H_{2}, H_{4}, P_{1}, P_{2}, S, Q_{i}, i=1, \ldots, 7, W_{i}, i=1, \ldots, 4$, $\Gamma_{1}=\operatorname{diag}\left(\gamma_{11}, \ldots, \gamma_{1 n}\right)$, and $\Gamma_{2}=\operatorname{diag}\left(\gamma_{21}, \ldots, \gamma_{2 n}\right)$, such that, for any appropriate dimensions constant matrices $H_{1}, H_{3}, X, Y$, the following LMIs hold:

$$
\begin{gathered}
\left(\begin{array}{cc}
W_{2} & X \\
X & W_{2}
\end{array}\right) \geq 0, \quad\left(\begin{array}{cc}
W_{4} & Y \\
Y & W_{4}
\end{array}\right) \geq 0, \\
\Omega_{1}^{\prime}+\Omega_{2}^{\prime}+L_{3}^{T}\left[e^{-\lambda \tau_{2}} Q_{2}+e^{-\lambda \tau_{1}} Q_{3}\right] L_{3} \\
+L_{8}^{T}\left[e^{-\lambda \sigma_{2}} Q_{5}+e^{-\lambda \sigma_{1}} Q_{6}\right] L_{8}<0,
\end{gathered}
$$

where $\Omega_{1}^{\prime}=\operatorname{diag}\left(\lambda P_{1}+Q_{1}-e^{-\lambda \tau_{1}} W_{1}-H_{1}^{T} A-A^{T} H_{1}, e^{-\lambda \tau_{1}}\left(Q_{1}-\right.\right.$ $\left.Q_{2}\right)-e^{-\lambda \tau_{1}} W_{1}-e^{-\lambda \tau_{2}} W_{2}, e^{-\lambda \tau_{1}}\left(1-\tau_{4}\right) Q_{3}-e^{-\lambda \tau_{2}}\left[\left(1-\tau_{3}\right) Q_{2}+\right.$ $\left.2 W_{2}-X^{T}-X\right],-e^{-\lambda \tau_{2}} W_{2}, \tau_{1}^{2} W_{1}+\left(\tau_{2}-\tau_{1}\right)^{2} W_{2}-H_{2}^{T}-H_{2}$, $\lambda P_{2}+Q_{4}-e^{-\lambda \sigma_{1}} W_{3}-2 M_{1}^{T} \Gamma_{1} M_{0}-H_{3}^{T} C-C^{T} H_{3},-e^{-\lambda \sigma_{1}}\left(Q_{4}-\right.$ $\left.Q_{5}+W_{3}\right)-e^{-\lambda \sigma_{2}} W_{4}, e^{-\lambda \sigma_{1}}\left(1-\sigma_{4}\right) Q_{6}-e^{-\lambda \sigma_{2}}\left[\left(1-\sigma_{3}\right) Q_{5}+\right.$ $\left.2 W_{4}-Y^{T}-Y\right]-M_{1}^{T} \Gamma_{2} M_{0},-e^{-\lambda \sigma_{2}}\left(Q_{4}+W_{4}\right), \sigma_{1}^{2} W_{3}+\left(\sigma_{2}-\right.$ $\left.\sigma_{1}\right)^{2} W_{4}-H_{4}^{T}-H_{4}, Q_{7}-2 \Gamma_{1}+\vartheta_{1}^{2} S,-e^{-\lambda \sigma_{2}} Q_{7}\left(1-\sigma_{4}\right)-2 \Gamma_{2}$, $\left.-e^{-\lambda \vartheta_{1}} S,-e^{-\lambda \vartheta_{1}} S\right), \Omega_{2}^{\prime}=\operatorname{sym}\left(L_{1}^{T} e^{-\lambda \tau_{1}} W_{1} L_{2}+L_{1}^{T}\left[P_{1}-H_{1}^{T}-\right.\right.$ $\left.H_{2}^{T} A\right] L_{5}+I_{1}^{T} H_{1}^{T} B L_{12}+L_{2}^{T} e^{-\lambda \tau_{2}}\left(W_{2}-X\right) L_{3}+L_{2}^{T} e^{-\lambda \tau_{2}} X L_{4}+$ $L_{3}^{T} e^{-\lambda \tau_{2}}\left(W_{2}-X\right) L_{4}+L_{3}^{T} H_{3}^{T} D L_{6}+L_{3}^{T} H_{4}^{T} D L_{10}+I_{5}^{T} H_{2}^{T} B L_{12}+$ $L_{6}^{T} e^{-\lambda \sigma_{1}} W_{3} L_{7}+L_{6}^{T}\left[P_{2}-H_{3}^{T}-H_{4}^{T} C\right] L_{10}+L_{6}^{T}\left[\Gamma_{1} M_{0}+M_{1}^{T} \Gamma_{1}\right] L_{11}$ $+L_{7}^{T} e^{-\lambda \sigma_{2}}\left(W_{4}-Y\right) L_{8}+L_{7}^{T} e^{-\lambda \sigma_{2}} Y L_{9}+L_{8}^{T} e^{-\lambda \sigma_{2}}\left(W_{4}-Y\right) L_{9}+$ $\left.L_{8}^{T}\left[\Gamma_{2} M_{0}+M_{1}^{T} \Gamma_{2}\right] L_{12}\right)$, and $L_{i}=\left[0_{n \times(i-1) n}, I_{n \times n}, 0_{n \times(15-i) n}\right], i=$ $1, \ldots, 12$.

Proof. Based on system (14), we construct the following Lyapunov-Krasovskii functional:

$$
\begin{aligned}
V(x(t), y(t))= & V_{1}(x(t), y(t))+V_{2}(x(t), y(t)) \\
& +V_{3}(x(t), y(t))+V_{4}(x(t), y(t)),
\end{aligned}
$$

where $V_{1}(x(t), y(t)), V_{2}(x(t), y(t))$, and $V_{3}(x(t), y(t))$ are defined as in Theorem 8 and

$$
\begin{aligned}
V_{4}(x(t), y(t)) \\
\quad=\int_{-\vartheta_{1}}^{0} \int_{t+\theta}^{t} \vartheta_{1} e^{\lambda(s-t)} g^{T}(y(s)) S g(y(s)) d s d \theta .
\end{aligned}
$$

Taking the derivative of $V_{4}$,

$$
\begin{aligned}
\dot{V}_{4}(x(t), y(t)) & \\
\leq & -\lambda \int_{-\vartheta_{1}}^{0} \int_{t+\theta}^{t} \vartheta_{1} e^{\lambda(s-t)} g^{T}(y(s)) S g(y(s)) d s d \theta \\
& +\vartheta_{1}^{2} g^{\mathrm{T}}(y(t)) S g(y(t)) \\
& -e^{-\lambda \vartheta_{1}} \int_{t-\vartheta_{1}}^{t} \vartheta_{1} g^{T}(y(\theta)) S g(y(\theta)) d \theta .
\end{aligned}
$$


By Lemma 6, we get that

$$
\begin{aligned}
& \dot{V}_{4}(x(t), y(t)) \\
& \leq-\lambda \int_{-\vartheta_{1}}^{0} \int_{t+\theta}^{t} \vartheta_{1} e^{\lambda(s-t)} g^{T}(y(s)) S g(y(s)) d s d \theta \\
& +\vartheta_{1}^{2} g^{T}(y(t)) S g(y(t))-e^{-\lambda \vartheta_{1}} \frac{\vartheta_{1}}{\vartheta_{1}-\vartheta(t)} \\
& \times\left(\int_{t-\vartheta_{1}}^{t-\vartheta(t)} g(y(\theta)) d \theta\right)^{T} S\left(\int_{t-\vartheta_{1}}^{t-\vartheta(t)} g(y(\theta)) d \theta\right) \\
& -e^{-\lambda \vartheta_{1}} \frac{\vartheta_{1}}{\vartheta(t)} \\
& \times\left(\int_{t-\vartheta(t)}^{t} g(y(\theta)) d \theta\right)^{T} S\left(\int_{t-\vartheta(t)}^{t} g(y(\theta)) d \theta\right) \\
& \leq-\lambda \int_{-\vartheta_{1}}^{0} \int_{t+\theta}^{t} \vartheta_{1} e^{\lambda(s-t)} g^{T}(y(s)) S g(y(s)) d s d \theta \\
& +\vartheta_{1}^{2} g^{T}(y(t)) S g(y(t))-e^{-\lambda \vartheta_{1}}\left(1+\frac{\vartheta(t)}{\vartheta_{1}}\right) \\
& \times\left(\int_{t-\vartheta_{1}}^{t-\vartheta(t)} g(y(\theta)) d \theta\right)^{T} S\left(\int_{t-\vartheta_{1}}^{t-\vartheta(t)} g(y(\theta)) d \theta\right) \\
& -e^{-\lambda \vartheta_{1}}\left(1+\frac{\vartheta_{1}-\vartheta(t)}{\vartheta_{1}}\right) \\
& \times\left(\int_{t-\vartheta(t)}^{t} g(y(\theta)) d \theta\right)^{T} S\left(\int_{t-\vartheta(t)}^{t} g(y(\theta)) d \theta\right) .
\end{aligned}
$$

Combining (23)-(31), (42), and (45), we get

$$
\begin{gathered}
\dot{V}(x(t), y(t))+\lambda V(x(t), y(t)) \\
\leq \xi_{1}^{T}(t)\left\{\Omega+L_{3}^{T}\left[e^{-\lambda \tau_{2}}\left(\dot{\tau}(t)-\tau_{3}\right) Q_{2}\right.\right. \\
\left.+e^{-\lambda \tau_{1}}\left(\tau_{4}-\dot{\tau}(t)\right) Q_{3}\right] L_{3} \\
+L_{8}^{T}\left[e^{-\lambda \sigma_{2}}\left(\dot{\sigma}(t)-\sigma_{3}\right) Q_{5}\right. \\
\left.\left.+e^{-\lambda \sigma_{1}}\left(\sigma_{4}-\dot{\sigma}(t)\right) Q_{6}\right] L_{8}\right\} \xi_{1}(t),
\end{gathered}
$$

where

$$
\begin{aligned}
\xi_{1}^{T}(t)=\left[x^{T}(t), x^{T}\left(t-\tau_{1}\right), x^{T}(t-\tau(t)),\right. \\
\quad x^{T}\left(t-\tau_{2}\right), \dot{x}^{T}(t), y^{T}(t), y^{T}\left(t-\sigma_{1}\right), \\
y^{T}(t-\sigma(t)), y^{T}\left(t-\sigma_{2}\right), \dot{y}^{T}(t), g^{T}(y(t)),
\end{aligned}
$$

$$
\begin{aligned}
& g^{T}(y(t-\sigma(t))),\left(\int_{t-\vartheta_{1}}^{t-\vartheta(t)} g(y(\theta)) d \theta\right)^{T}, \\
& \left.\left(\int_{t-\vartheta(t)}^{t} g(y(\theta)) d \theta\right)^{T}\right] .
\end{aligned}
$$

By (40) and (41), we get that $\dot{V}(x(t), y(t))+\lambda V(x(t), y(t))<0$. Similar to the proof of Theorem 8 we have

$$
\|z(t)\|^{2} \leq \frac{\lambda_{2}^{\prime}}{\lambda_{1}} e^{-\lambda\left(t-t_{0}\right)}\left\|z\left(t_{0}\right)\right\|_{C_{1}}^{2} .
$$

where $\lambda_{2}^{\prime}=\lambda_{2}+(1 / 2) \vartheta_{1}^{3} m^{2} \lambda_{\max }(S)$ and $\lambda_{1}, \lambda_{2}$ are defined as Theorem 8 . Let $\alpha^{\prime}=\left(\lambda_{2}^{\prime} / \lambda_{1}\right)^{1 / 2}$, and, by Definition 5 , the genetic regulatory network (14) is exponentially stable.

The proof is completed.

Remark 10. In the proof of Theorems 8 and 9, we use convex combination and interactive convex combination definition to estimate the upper bound of derivative function of the Lyapunov-Krasovskii functional and obtain some new conservative weaker sufficient conditions.

Remark 11. When the lower bound of derivatives of time-varying delays is immeasurable, let $\int_{t-\tau_{2}}^{t-\tau(t)} e^{\lambda(s-t)} x^{T}(s) Q_{3} x(s) d s=0$ and $\int_{t-\sigma_{2}}^{t-\sigma(t)} e^{\lambda(s-t)} y^{T}$ $(s) Q_{6} y(s) d s=0$ in Theorem 8 (20) or Theorem 9 (41); our results are true still.

\section{Numerical Examples}

In this section, two examples are given to illustrate the effectiveness of our theoretical results.

Example 1. Consider a genetic regulatory network model reported by Elowitz and Leiber [24], which studied the dynamics of repressilator which is cyclic negative-feedback loop comprising three repressor genes (lacl, tet $R$, and $c l$ ) and their promoters $(\mathrm{cl}$, lacl, and tet $R)$ :

$$
\begin{gathered}
\frac{d x_{i}}{d t}=-x_{i}+\frac{\alpha}{1+y_{j}^{n}}+\alpha_{0}, \\
\frac{d y_{i}}{d t}=\beta\left(x_{i}-y_{i}\right) .
\end{gathered}
$$

Taking time-varying delays into account and shifting the equilibrium point to the origin, one gets the following model:

$$
\begin{gathered}
\dot{x}(t)=-A x(t)+B g(y(t-\sigma(t))), \\
\dot{y}(t)=-C y(t)+D x(t-\tau(t)),
\end{gathered}
$$

where $A=\operatorname{diag}(2,2,2), C=\operatorname{diag}(3,3,3), D=\operatorname{diag}(1,1,1)$, and the coupling matrix

$$
B=1.5 \times\left(\begin{array}{ccc}
0 & 0 & -1 \\
-1 & 0 & 0 \\
0 & -1 & 0
\end{array}\right) .
$$




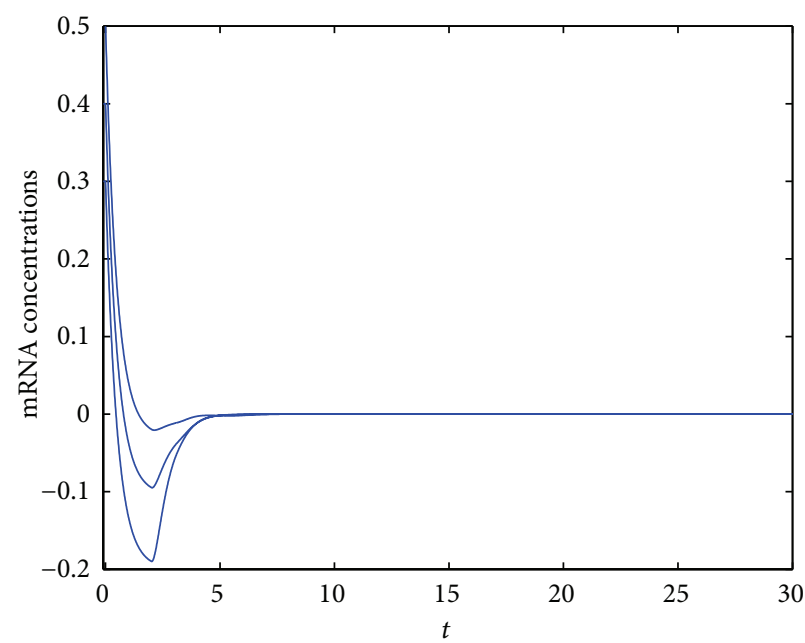

(a)

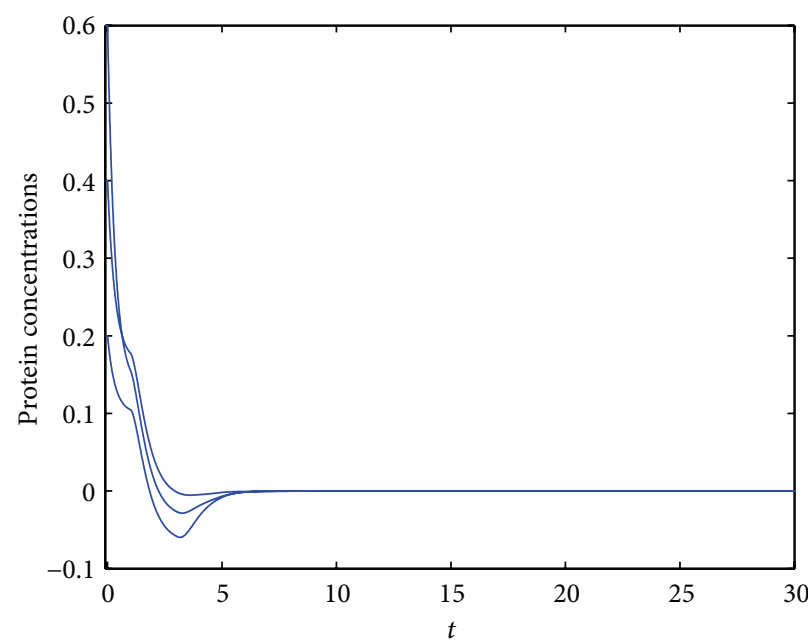

(b)

Figure 1: (a) mRNA concentrations $x(t)$. (b) Protein concentrations $y(t)$.

The gene regulation function is taken as $g(x)=x^{2} /(1+$ $\left.x^{2}\right), M_{0}=\operatorname{diag}(0,0,0)$, and $M_{1}=\operatorname{diag}(0.65,0.65,0.65)$. The time delays $\sigma(t)$ and $\tau(t)$ are assumed to be

$$
\sigma(t)=1+2 \sin ^{2} t, \quad \tau(t)=1+\cos ^{2} t .
$$

We can get the parameters as follows:

$$
\begin{array}{llll}
\sigma_{1}=1, & \sigma_{2}=3, & \sigma_{3}=-2, & \sigma_{4}=2, \\
\tau_{1}=1, & \tau_{2}=2, & \tau_{3}=-1, & \tau_{4}=1,
\end{array}
$$

and choose $\lambda=0.5$. In accordance with the condition in Theorem 8, system (50) is exponentially stable. By using the MATLAB LMI toolbox, we can get the feasible solutions. Due to the space limitation, we only list matrices $P_{1}$ and $P_{2}$ here as follows:

$$
\begin{aligned}
& P_{1}=\left(\begin{array}{ccc}
17.1041 & 7.9997 & 7.9997 \\
7.9997 & 17.1041 & 7.9997 \\
7.9997 & 7.9997 & 17.1041
\end{array}\right), \\
& P_{2}=\left(\begin{array}{ccc}
13.8943 & 0.5573 & 0.5573 \\
0.5573 & 13.8943 & 0.5573 \\
0.5573 & 0.5573 & 13.8943
\end{array}\right) .
\end{aligned}
$$

The initial condition is $x(0)=(0.3,0.5,0.4)^{T}$ and $y(0)=$ $(0.2,0.4,0.6)^{T}$. The simulation results of the trajectories are shown in Figure 1.

Example 2. In this example, we consider the genetic regulatory network (14) with time-varying delays and continuous distributed delays, in which the parameters are listed as follows:

$$
\begin{gathered}
A=\operatorname{diag}(1,2,3), \quad C=\operatorname{diag}(5,4,5), \\
D=\operatorname{diag}(0.3,0.2,0.4), \quad B=\left(\begin{array}{ccc}
0 & 0.8 & 0 \\
0 & 0 & 0.8 \\
0.8 & 0 & 0
\end{array}\right),
\end{gathered}
$$

and $g(x)=x^{2} /\left(1+x^{2}\right), M_{0}=\operatorname{diag}(0,0,0)$, and $M_{1}=$ $\operatorname{diag}(0.65,0.65,0.65)$. The time delays $\sigma(t), \tau(t)$, and $\vartheta(t)$ are assumed to be

$$
\begin{gathered}
\sigma(t)=0.5+0.3 \sin ^{2} t, \quad \tau(t)=0.4+0.1 \cos ^{2} t, \\
\vartheta(t)=2 \sin ^{2}(t) .
\end{gathered}
$$

We can get the parameters as follows:

$$
\begin{gathered}
\sigma_{1}=0.5, \quad \sigma_{2}=0.8, \quad \sigma_{3}=-0.3, \quad \sigma_{4}=0.3, \\
\tau_{1}=0.4, \quad \tau_{2}=0.5, \quad \tau_{3}=-0.1, \\
\tau_{4}=0.1, \quad \vartheta_{1}=2
\end{gathered}
$$

and choose $\lambda=0.2$. By using the MATLAB LMI toolbox, we can get the feasible solutions. Due to the space limitation, we only list matrices $P_{1}$ and $P_{2}$ here as follows:

$$
\begin{gathered}
P_{1}=\left(\begin{array}{ccc}
7.1735 & -0.4713 & -0.2152 \\
-0.4713 & 7.2143 & -0.3071 \\
-0.2152 & -0.3071 & 6.9607
\end{array}\right), \\
P_{2}=\left(\begin{array}{ccc}
7.9363 & 0 & 0 \\
0 & 8.0199 & 0 \\
0 & 0 & 7.9001
\end{array}\right) .
\end{gathered}
$$

\section{Concluding Remarks}

This paper has investigated the exponential stability of genetic regulatory networks with time-varying delays and continuous distributed delays. By using the novel LyapunovKrasovskii functions and employing the Jensen inequality and the interactive convex combination method, some sufficient criteria are given to ensure the exponential stability with less conservative. All the obtained conditions are dependent on the delays and on linear matrix inequalities. Two examples are provided to illustrate the effectiveness of our results. 


\section{Conflict of Interests}

The authors declare that there is no conflict of interests regarding the publication of this paper.

\section{Acknowledgments}

This work was supported by the National Natural Science Foundations of China (61273084, 61233014, and 61174217), the Natural Science Foundation for Distinguished Young Scholar of Shandong Province of China (JQ200919), the Independent Innovation Foundation of Shandong University (2012JC014), the Natural Science Foundation of Shandong Province of China (ZR2010AL016, ZR2011AL007), and the Doctoral Foundation of University of Jinan (XBS1244).

\section{References}

[1] H. Li and X. Yang, "Asymptotic stability analysis of genetic regulatory networks with time-varying delay," in Proceedings of the Chinese Control and Decision Conference (CCDC '10), pp. 566-571, May 2010.

[2] Y. Tang, Z. Wang, and J.-A. Fang, "Parameters identification of unknown delayed genetic regulatory networks by a switching particle swarm optimization algorithm," Expert Systems with Applications, vol. 38, no. 3, pp. 2523-2535, 2011.

[3] S. Huang, "Gene expression profiling, genetic networks, and cellular states: an integrating concept for tumorigenesis and drug discovery," Journal of Molecular Medicine, vol. 77, no. 6, pp. 469-480, 1999.

[4] S. A. Kauffman, "Metabolic stability and epigenesis in randomly constructed genetic nets," Journal of Theoretical Biology, vol. 22, no. 3, pp. 437-467, 1969.

[5] R. Somogyi and C. A. Sniegoski, "Modeling the complexity of genetic networks: understanding multigenic and pleiotropic regulation," Complexity, vol. 1, no. 6, pp. 45-63, 1996.

[6] R. Thomas, "Boolean formalization of genetic control circuits," Journal of Theoretical Biology, vol. 42, no. 3, pp. 563-585, 1973.

[7] F. Ren and J. Cao, "Asymptotic and robust stability of genetic regulatory networks with time-varying delays," Neurocomputing, vol. 71, no. 4-6, pp. 834-842, 2008.

[8] M. de Hoon, S. Imoto, K. Kobayashi, N. Ogasawara, and S. Miyano, "Inferring gene regulatory networks fromtime-ordered gene expression data of bacillus subtilis using differential equations," in Proceedings Pacific Symposiumon Biocomputing, vol. 8, pp. 17-28, 2003.

[9] L. Chen and K. Aihara, "Stability of genetic regulatory networks with time delay," IEEE Transactions on Circuits and Systems I: Fundamental Theory and Applications, vol. 49, no. 5, pp. 602608, 2002.

[10] W. Zhang, J.-A. Fang, and Y. Tang, "New robust stability analysis for genetic regulatory networks with random discrete delays and distributed delays," Neurocomputing, vol. 74, no. 14-15, pp. 2344-2360, 2011.

[11] X. Lou, Q. Ye, and B. Cui, "Exponential stability of genetic regulatory networks with random delays," Neurocomputing, vol. 73, no. 4-6, pp. 759-769, 2010.

[12] W. Wang and S. Zhong, "Delay-dependent stability criteria for genetic regulatory networks with time-varying delays and nonlinear disturbance," Communications in Nonlinear Science and Numerical Simulation, vol. 17, no. 9, pp. 3597-3611, 2012.
[13] R. Rakkiyappan and P. Balasubramaniam, "Delay-probabilitydistribution-dependent stability of uncertain stochastic genetic regulatory networks with mixed time-varying delays: an LMI approach," Nonlinear Analysis: Hybrid Systems, vol. 4, no. 3, pp. 600-607, 2010.

[14] W. Wang and S. Zhong, "Stochastic stability analysis of uncertain genetic regulatory networks with mixed time-varying delays," Neurocomputing, vol. 82, no. 1, pp. 143-156, 2012.

[15] J. Cao and F. Ren, "Exponential stability of discrete-time genetic regulatory networks with delays," IEEE Transactions on Neural Networks, vol. 19, no. 3, pp. 520-523, 2008.

[16] F. Wu, "Stability analysis of genetic regulatory networks with multiple time delays," in Proceedings of the 29th Annual International Conference of the IEEE EMBS Cite Internationale, Lyon, France, 2007.

[17] W. Zhang, J.-A. Fang, and Y. Tang, "Stochastic stability of Markovian jumping genetic regulatory networks with mixed time delays," Applied Mathematics and Computation, vol. 217, no. 17, pp. 7210-7225, 2011.

[18] W. Zhang, Y. Tang, J. Fang, and X. Wu, "Stochastic stability of genetic regulatory networks with a finite set delay characterization," Chaos, vol. 2, no. 22, Article ID 023106, 2012.

[19] G. Wang and J. Cao, "Robust exponential stability analysis for stochastic genetic networks with uncertain parameters," Communications in Nonlinear Science and Numerical Simulation, vol. 14, no. 8, pp. 3369-3378, 2009.

[20] C.-H. Yuh, H. Bolouri, and E. H. Davidson, "Genomic cisregulatory logic: experimental and computational analysis of a sea urchin gene," Science, vol. 279, no. 5358, pp. 1896-1902, 1998.

[21] C. Li, L. Chen, and K. Aihara, "Stability of genetic networks with SUM regulatory logic: Lur'e system and LMI approach," IEEE Transactions on Circuits and Systems I: Regular Papers, vol. 53, no. 11, pp. 2451-2458, 2006.

[22] W. Su and Y. Chen, "Global robust stability criteria of stochastic Cohen-Grossberg neural networks with discrete and distributed time-varying delays," Communications in Nonlinear Science and Numerical Simulation, vol. 14, no. 2, pp. 520-528, 2009.

[23] P. Park, J. W. Ko, and C. Jeong, "Reciprocally convex approach to stability of systems with time-varying delays," Automatica, vol. 47, no. 1, pp. 235-238, 2011.

[24] M. B. Elowitz and S. Leibier, "A synthetic oscillatory network of transcriptional regulators," Nature, vol. 403, no. 1, pp. 335-338, 2000. 


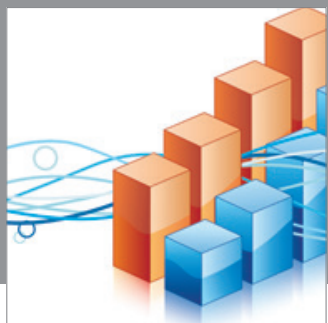

Advances in

Operations Research

mansans

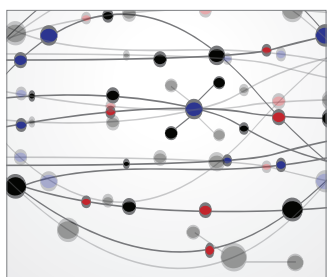

The Scientific World Journal
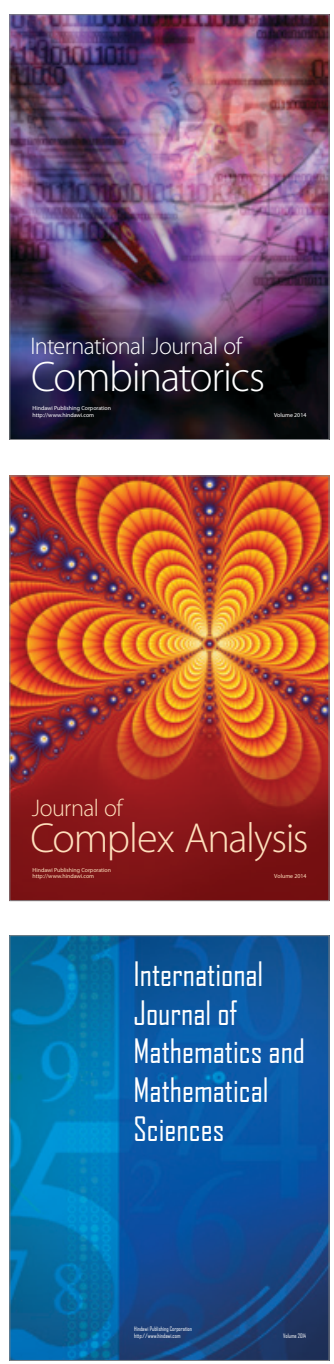
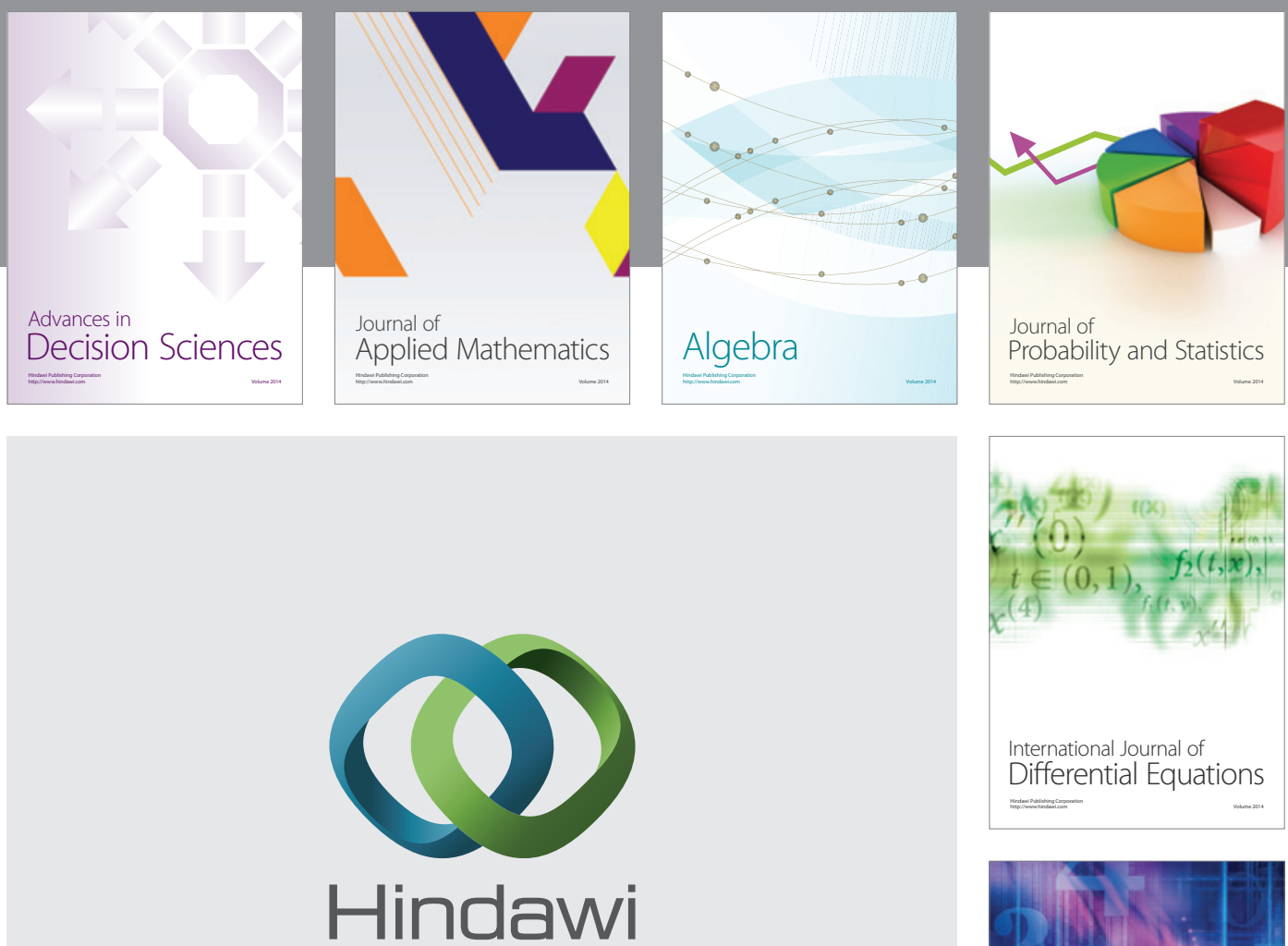

Submit your manuscripts at http://www.hindawi.com
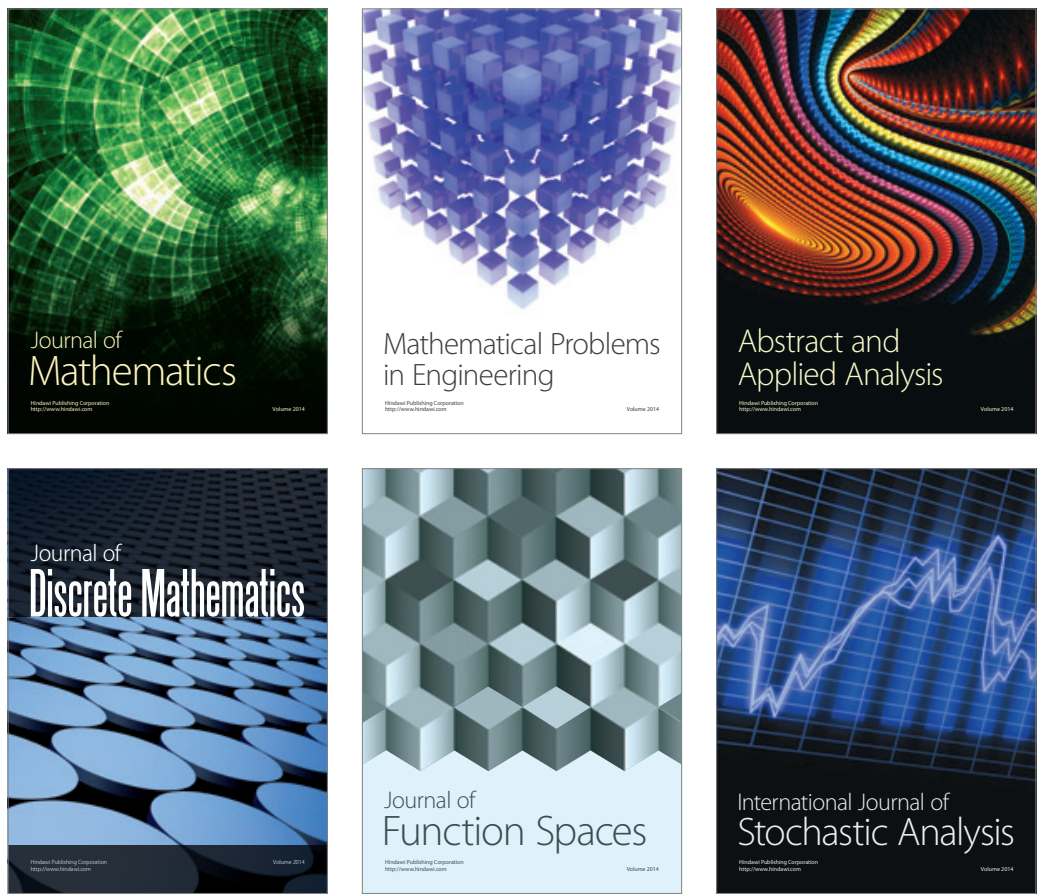

Journal of

Function Spaces

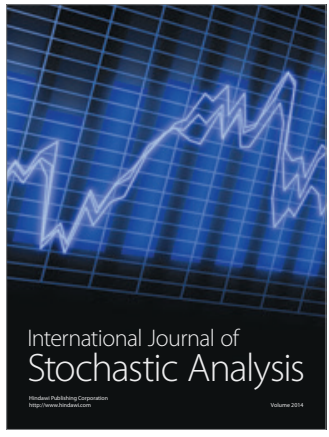

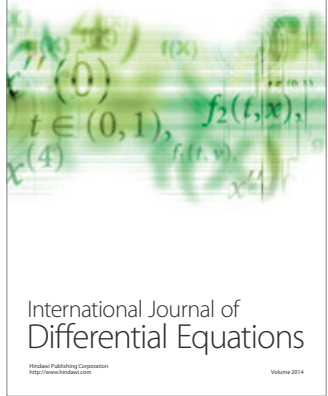
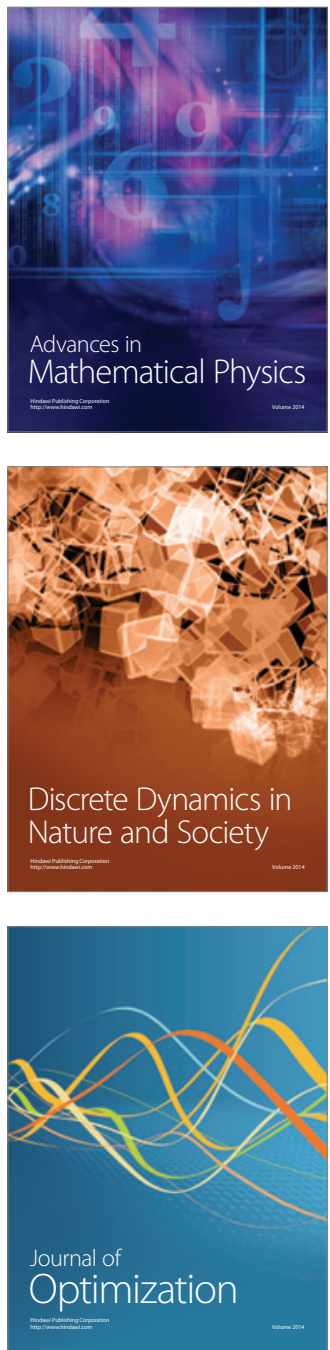